\title{
A surface spherical harmonic expansion of gravity anomalies on the ellipsoid
}

\author{
S. J. Claessens • C. Hirt
}

Received: date / Accepted: date

\begin{abstract}
A surface spherical harmonic expansion of gravity anomalies with respect to a geodetic reference ellipsoid can be used to model the global gravity field and reveal its spectral properties. In this paper, a direct and rigorous transformation between solid spherical harmonic coefficients of the Earth's disturbing potential and surface spherical harmonic coefficients of gravity anomalies in ellipsoidal approximation with respect to a reference ellipsoid is derived. This transformation cannot rigorously be achieved by the HotineJekeli transformation between spherical and ellipsoidal harmonic coefficients. The method derived here is used to create a surface spherical harmonic model of gravity anomalies with respect to the GRS80 ellipsoid from the EGM2008 global gravity model. Internal validation of the model shows a global RMS precision of $<1$ nanoGal. This is significantly more precise than previous solutions based on spherical approximation or approximations to order $e^{2}$ or $e^{3}$, which are shown to be insufficient for the generation of surface spherical harmonic coefficients with respect to a geodetic reference ellipsoid. Numerical results of two applications of the new method (the computation of ellipsoidal corrections to gravimetric geoid computation, and area means of gravity anomalies in ellipsoidal approximation) are provided.
\end{abstract}

Keywords surface spherical harmonic expansion . solid spherical harmonic expansion · gravity anomalies · global gravity model · ellipsoidal corrections

S. J. Claessens · C. Hirt

Western Australian Geodesy Group and The Institute for Geoscience Research, Curtin University, GPO Box U1987,

Perth, WA, 6845, Australia

Tel.: +61 892663505

Fax: +61 892662703

E-mail: s.claessens@curtin.edu.au

\section{Introduction}

A surface spherical harmonic expansion is an expansion of any function on a star-shaped surface, i.e., a surface where each point on the surface is uniquely defined by its geocentric latitude and longitude (e.g., Jekeli 1988, Grafarend and Engels 1994). A surface spherical harmonic expansion is distinctly different from a solid spherical harmonic expansion, which is routinely employed in global gravity models. Most importantly, it is restricted to the representation of a function on a two-dimensional surface instead of in three-dimensional space. Other than in a solid harmonic expansion, the function to be expanded does not need to be harmonic. In addition, a surface harmonic expansion does not necessarily need to utilise spherical polar coordinates, although spherical polar coordinates are used here. A more detailed explanation of the differences between solid and surface spherical harmonic expansions is provided in Jekeli (1988).

A surface spherical harmonic expansion of gravity anomalies with respect to the geodetic reference ellipsoid (i.e. an oblate ellipsoid of revolution) can be used to study the spectral properties of the global gravity anomaly field. It is used in various applications. For example, the spectral form of Stokes's formula for geoid computation contains a surface spherical harmonic series of gravity anomalies (e.g., Heiskanen and Moritz 1967). Therefore, surface harmonic coefficients of gravity anomalies are used in the study of Stokesian geoid computation schemes, such as the study of Stokes kernel modification (e.g., Vaníček and Featherstone 1998, Featherstone et al. 1998, Sjöberg and Featherstone 2004), and the derivation of ellipsoidal corrections to a Stokesian geoid computation (e.g., Heck and Seitz 2003, Claessens 2006). It is shown here that a surface harmonic expan- 
sion of gravity anomalies can also be used to efficiently compute area means of gravity anomalies from a global gravity model (e.g. Hirt and Claessens 2011), which are required in a combined gravimetric geoid solution (e.g., Featherstone et al. 2011; Claessens et al. 2011).

A surface spherical harmonic expansion of gravity anomalies can be computed via a spherical harmonic analysis from a grid of gravity anomalies, which can be created from a global gravity model through spherical harmonic synthesis. This synthesis-analysis procedure suffers from discretisation errors (e.g., Colombo 1981, Albertella et al. 1993). This paper proposes an alternative procedure based on a direct transformation of spherical harmonic coefficients of the Earth's disturbing potential given by a global gravity model, avoiding discretisation errors. Apart from the applications mentioned above, it can therefore also be used to estimate the discretisation errors and validate spherical harmonic analysis and synthesis software.

The transformation between spherical harmonic coefficients of the disturbing potential and gravity anomalies is well-known in spherical and constant radius (SCR) approximation (e.g., Heiskanen and Moritz 1967). The transformation in ellipsoidal approximation was investigated by, e.g., Cruz (1986), and Heck and Seitz (2003), but their methods were not intended for transformation of high-degree coefficients and are of insufficient precision for coefficients beyond degree $\sim 180$. The derivation presented here is theoretically exact in ellipsoidal approximation without the need for any further approximations. The validity of the method up to high degree and order $(\sim 2160)$ is verified numerically, and two applications are shown.

An alternative to the surface spherical harmonic expansion is an ellipsoidal harmonic expansion. Transformation between spherical and ellipsoidal harmonic coefficients is possible (e.g. Buchdahl et al. 1977, Jekeli 1988), and in geodesy this procedure is called a HotineJekeli transformation (Sebera et al. 2012). However, the Hotine-Jekeli transformation cannot directly be applied to compute a harmonic expansion of gravity anomalies with respect to an ellipsoid (see section 5). A rigorous transformation between disturbing potential and gravity anomalies in the ellipsoidal spectral domain can be found using a different approach, by applying a technique described in Claessens and Featherstone (2008). This is shown in sections 2 and 3 , and the method is numerically validated in section 4 . An overview of similarities and differences between the method presented here and the Hotine-Jekeli transformation is presented in section 5. Some applications are presented in section 6.

\section{Spherical harmonic expansion of gravity anomalies}

We consider here the solid gravity anomaly $\Delta g$ (Vaníček et al. 2004), which is defined by the fundamental gravimetric equation

$\Delta g=-\frac{\partial T}{\partial h}+\frac{1}{\gamma} \frac{\partial \gamma}{\partial h} T$

where $T$ is the disturbing potential, $\gamma$ is the magnitude of reference gravity and both partial derivatives are with respect to the ellipsoidal normal. This solid gravity anomaly can be viewed as a $3 \mathrm{D}$ functional which can be evaluated at any height above the ellipsoid (e.g., Barthelmes 2009). Here, gravity anomalies on the surface of the ellipsoid are used, i.e., the functionals $T$, $\gamma$ and their derivatives are evaluated on the ellipsoid. The classical definition of the gravity anomaly utilised in Stokes's theory (magnitude of gravity on the geoid minus magnitude of reference gravity at the ellipsoid) follows from this solid gravity anomaly with negligible approximation errors (e.g., Moritz 1989, Vaníček et al. 2004).

The spherical harmonic expansion most frequently used in geodesy is the solid spherical harmonic expansion, which is routinely employed in global gravity models. Solid spherical harmonic expansions can be used to represent a function in three-dimensional space, that is harmonic on and outside a sphere of certain radius, such as the Earth's disturbing potential $T$

$T(r, \theta, \lambda)=\sum_{n=0}^{\infty}\left(\frac{R}{r}\right)^{n+1} \sum_{m=-n}^{n} \bar{T}_{n m}^{R} \bar{Y}_{n m}(\theta, \lambda)$

where $(r, \theta, \lambda)$ are spherical polar coordinates, $\bar{T}_{n m}^{R}$ are the solid spherical harmonic coefficients of degree $n$ and order $m, \bar{Y}_{n m}$ are the spherical harmonic functions, and $R$ is the radius of some reference sphere. The overbars indicate that the spherical harmonic functions are fully

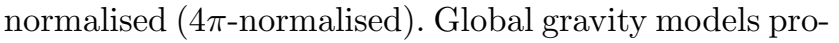
vide coefficients of the gravitational potential, but these can easily be transformed into the coefficients of the disturbing potential $\bar{T}_{n m}^{R}$ by subtracting the potential implied by the reference field (e.g., Smith 1998).

Unlike the Earth's disturbing potential, gravity anomalies as defined in Eq. (1) are not harmonic, and thus cannot be represented by a solid spherical harmonic series. They can, however, be represented by a set of surface spherical harmonic coefficients, also called a Laplace surface harmonic expansion

$\Delta g(\theta, \lambda)=\sum_{n=0}^{\infty} \sum_{m=-n}^{n} \overline{\Delta g}_{n m}^{\mathrm{e}} \bar{Y}_{n m}(\theta, \lambda)$ 
where $\overline{\Delta g}_{n m}^{\mathrm{e}}$ are surface spherical harmonic coefficients of the gravity anomalies. Since an ellipsoid is a starshaped surface, any function on the ellipsoid can be expanded into a series of surface spherical harmonic coefficients. The superscript e in the coefficients in Eq. (3) indicates that this series is defined with respect to an ellipsoid. Note that these surface spherical harmonic coefficients with respect to an ellipsoidal surface are not, in general, equal to ellipsoidal harmonic coefficients, because ellipsoidal harmonic coefficients must utilise ellipsoidal coordinates instead of spherical polar coordinates.

If gravity anomalies are given on the surface of an ellipsoid, the coefficients $\overline{\Delta g}_{n m}^{\mathrm{e}}$ can be computed via an integration over the unit sphere (e.g., Heiskanen and Moritz 1967, Eq. 1-76), which in practice must be discretised (e.g., Hirt et al. 2011). Here, a direct relation between $\overline{\Delta g}_{n m}^{\mathrm{e}}$ and $\bar{T}_{n m}^{R}$ is sought.

\section{Spectral relation between disturbing potential and gravity anomaly}

\subsection{Spherical and constant radius approximation}

Equation (1) gives the relation between the disturbing potential $T$ and the gravity anomaly $\Delta g$ in the space domain. This relation is known to be simpler in the spectral domain in spherical and constant radius (SCR) approximation (e.g., Heiskanen and Moritz 1967, p. 89)

$\overline{\Delta g}_{n m}^{\mathrm{e}} \approx \frac{n-1}{R} \bar{T}_{n m}^{R}$

The spherical approximation that this relation relies on consists of two steps. Firstly, the normal derivative to the ellipsoid is approximated by the radial derivative. This approximation is pre-dominantly of a shortwavelength nature, as it is roughly proportionate to the north-south vertical deflection (Claessens 2006). Secondly, the reference gravity $\gamma$ is approximated by an isotropic reference gravity $\widetilde{\gamma}$, which is simply the gravity induced by a point mass or a homogeneous sphere with a total mass equal to that of the Earth. This second approximation is pre-dominantly of a long-wavelength nature, as it is rougly proportionate to the height anomaly. The gravity anomaly in spherical approximation is thus

$\widetilde{\Delta g}=-\frac{\partial T}{\partial r}-\frac{2}{r_{\mathrm{e}}} T$

where the tilde over $\Delta g$ indicates that the spherical approximation is applied, and $r_{\mathrm{e}}$ is the ellipsoidal radius, i.e., the distance between the centre and the surface of the ellipsoid (which is dependent on latitude). The error in the gravity anomaly introduced by the spherical approximation is well-known to be of the order of the flattening $f$ of the ellipsoid $(\approx 0.003)$ (e.g., Heiskanen and Moritz 1967, Rummel 1985).

Inserting the spherical harmonic representations of the disturbing potential (Eq. (2)) and its radial derivative into Eq. (5) gives an expression for the gravity anomaly in terms of geopotential coefficients

$\widetilde{\Delta g}=\frac{1}{R} \sum_{n=0}^{\infty} \sum_{m=-n}^{n}(n-1)\left(\frac{R}{r_{\mathrm{e}}}\right)^{n+2} \bar{T}_{n m}^{R} \bar{Y}_{n m}$

The simple one-to-one relation between spherical harmonic coefficients in Eq. (4) only materialises when the ellipsoidal radius $r_{\mathrm{e}}$ is approximated by the radius of the reference sphere $R$, i.e., when the constant radius approximation is applied. The constant radius approximation has the largest effect on coefficients of high degree $n$, since for high degrees the term $\left(R / r_{\mathrm{e}}\right)^{n+2}$ is significantly different from 1.

Without the constant radius approximation, a simple one-to-one relation is not possible because $r_{\mathrm{e}}$ is a function of latitude. A solution can still be found using a procedure described in Claessens and Featherstone (2008). This involves expanding the term $\left(R / r_{\mathrm{e}}\right)^{n+2}$ into a binomial series

$\widetilde{\Delta g}=\frac{1}{R} \sum_{n=0}^{\infty} \sum_{m=-n}^{n}(n-1) \sum_{j=0}^{\infty} \alpha_{n+1, j} \sin ^{2 j} \theta \bar{T}_{n m}^{R} \bar{Y}_{n m}$

where

$\alpha_{n+1, j}=\left(\frac{R}{b}\right)^{n+2}(-1)^{j}\left(\begin{array}{c}\frac{n+2}{2} \\ j\end{array}\right) e^{2 j}$

In Eq. (8), $e^{2}$ is the square of the first numerical eccentricity of the ellipsoid, and it is assumed that the ellipsoid is an oblate ellipsoid of revolution. Since for any ellipsoid $0 \leq e^{2} \sin ^{2} \theta<1$, the binomial series will always be alternating and convergent.

A relation between surface spherical harmonic coefficients of gravity anomalies and solid spherical harmonic coefficients of the disturbing potential is only possible if all terms in Eq. (7), except the spherical harmonic functions $\bar{Y}_{n m}$, are independent of latitude and longitude. However, co-latitude $\theta$ appears in Eq. (7) outside the spherical harmonic function. This problem can be solved by shifting all dependence on $\theta$ in Eq. (7) into the spherical harmonic functions using a relation among spherical harmonic functions (Claessens 2005)

$\sin ^{2 j} \theta \bar{Y}_{n m}=\sum_{i=-j}^{j} \bar{K}_{n m}^{2 i, 2 j} \bar{Y}_{n+2 i, m}$

where spherical harmonic functions of negative degree should be set equal to zero. The weights $\bar{K}_{n m}^{2 i, 2 j}$ depend only on the spherical harmonic degree $n$, order $m$ and the summation indices $i$ and $j$. These weights can be 
computed most efficiently through an iterative scheme (Claessens 2005, Claessens and Featherstone 2008)

$\bar{K}_{n m}^{2 i, 2 j}=\sum_{k=-1}^{1} \bar{K}_{n m}^{2 k, 2} \bar{K}_{n+2 k, m}^{2(i-k), 2(j-1)}$

from the initial values given by

$$
\begin{aligned}
& \bar{K}_{n m}^{-2,2}=-\sqrt{\frac{\left[(n-1)^{2}-m^{2}\right]\left(n^{2}-m^{2}\right)}{(2 n-3)(2 n-1)^{2}(2 n+1)}} \\
& \bar{K}_{n m}^{0,2}=\frac{2\left(n^{2}+m^{2}+n-1\right)}{(2 n-1)(2 n+3)} \\
& \bar{K}_{n m}^{2,2}=-\sqrt{\frac{\left[(n+1)^{2}-m^{2}\right]\left[(n+2)^{2}-m^{2}\right]}{(2 n+1)(2 n+3)^{2}(2 n+5)}}
\end{aligned}
$$

These weights have also successfully been applied in the computation of the topographic potential generated by masses above a reference ellipsoid (Claessens and Hirt 2013).

Inserting Eq. (9) into Eq. (7) leads after comparison to Eq. (3) and a rearrangement of the summation indices to a relation between surface spherical harmonic coefficients of gravity anomalies and solid spherical harmonic coefficients of the disturbing potential in spherical approximation

$\widetilde{\overline{\Delta g}}_{n m}^{\mathrm{e}}=\sum_{i=-\infty}^{\infty} \widetilde{\lambda}_{n m i}^{\mathrm{g}} \bar{T}_{n-2 i, m}^{R}$

where

$\widetilde{\lambda}_{n m i}^{\mathrm{g}}=-\sum_{j=|i|}^{\infty} \frac{(n-1-2 i)}{R} \alpha_{n-2 i+1, j} \bar{K}_{n-2 i, m}^{2 i, 2 j}$

Here, again, the tildes indicate the use of the spherical approximation and the superscript $g$ indicates that the transformation is from disturbing potential to gravity anomaly. See Claessens (2006) for further details on the derivation of Eq. (12). This equation is the equivalent of Eq. (4), but without the constant radius approximation. Though much more complicated than Eq. (4), it still contains the spherical approximation. It is, however, possible to derive a similar relation without the need for the spherical approximation, as follows.

\subsection{Ellipsoidal approximation}

The spectral relation between disturbing potential and gravity anomalies on the ellipsoid was investigated by Cruz (1986), Heck and Seitz (2003) and others. All of these contributions rely on an approximation of Eq. (1) to the order of the first numerical eccentricity of the ellipsoid $e^{2}$, including an approximation of the geophysicalgeometric parameter $m$, a function of the Earth's angular velocity, by $e^{2} / 2$ based on a numerical coincidence
(Moritz 1989; see Sjöberg 2003 for a discussion). Here, it is shown that a solution can be found that avoids these approximations.

The derivation of the spectral relation between disturbing potential and gravity anomalies in spherical approximation (without constant radius approximation) presented in Section 3.1 relies on shifting the latitudedependent terms into the spherical harmonic functions $\bar{Y}_{n m}$ using a binomial expansion and Eq. (9). This same procedure can also be applied to the case in ellipsoidal approximation. This amounts to finding the spectral equivalent of Eq. (1), without further approximations.

The formula for the gravity anomaly on the righthand side of Eq. (1) consists of two terms: one term containing the normal derivative of the disturbing potential and one term containing the disturbing potential itself. These two terms can be treated separately. Claessens and Featherstone (2008) have derived the relation between solid spherical harmonic coefficients of the disturbing potential $\bar{T}_{n m}^{R}$ and surface spherical harmonic coefficients of its derivative with respect to the ellipsoidal normal $\overline{d h}_{h}^{\mathrm{e}}$

${\overline{d_{h} T}}_{n m}^{\mathrm{e}}=\sum_{i=-\infty}^{\infty} \lambda_{n m i}^{\mathrm{d}} \bar{T}_{n-2 i, m}^{R}$

where

$\lambda_{n m i}^{\mathrm{d}}=-\frac{1}{R} \sum_{j=|i|-1}^{\infty}\left[(n-2 i+1) \beta_{n-2 i, m i j}\right.$
$\left.+\gamma_{n-2 i, m i j}\right]$

$$
\begin{gathered}
\beta_{n m i j}=\bar{K}_{n m}^{2 i, 2 j} \sum_{k=0}^{j} \alpha_{n+1, j-k}(-1)^{k} e^{2 k} \\
\times \sum_{l=k-1}^{k}\left(2-e^{2}\right)^{l}\left(\begin{array}{c}
-\frac{1}{2} \\
l
\end{array}\right) \\
\gamma_{n m i j}=e^{2}\left[\sum_{p=i-1}^{i+1} \bar{K}_{n+2(i-p), m}^{2 p, 2 j} \bar{N}_{n, m, 2(i-p)}\right] \\
\times \sum_{k=0}^{j} \alpha_{n+1, j-k}(-1)^{k} e^{2 k}\left(2-e^{2}\right)^{k}\left(\begin{array}{c}
-\frac{1}{2} \\
k
\end{array}\right)
\end{gathered}
$$

and

$$
\begin{aligned}
& \bar{N}_{n, m,-2}=(n+1) \bar{K}_{n m}^{-2,2} \\
& \bar{N}_{n, m, 0}=\frac{3}{2} \bar{K}_{n m}^{0,2}-1 \\
& \bar{N}_{n, m, 2}=-n \bar{K}_{n m}^{2,2}
\end{aligned}
$$

Therefore, to find a relation for the gravity anomaly, only the second term on the right-hand side of Eq. (1) still needs to be evaluated. The procedure that will be followed here is the same as in the derivations above: the 
term $(1 / \gamma)(\partial \gamma / \partial h)$ is written as a power series in the squared sine of the geocentric co-latitude $\theta$, after which Eq. (9) is applied to move all dependence on latitude into the spherical harmonic functions.

According to Heiskanen and Moritz (1967, Eq. 2120), the normal derivative of the reference gravity $\gamma$ can be expressed as a function of the meridian radius of curvature $\rho$, the prime vertical radius of curvature $\nu$, and the angular velocity of the Earth's rotation $\omega$. Division of this formula by the reference gravity gives

$\frac{1}{\gamma} \frac{\partial \gamma}{\partial h}=-\frac{1}{\rho}-\frac{1}{\nu}-2 \frac{\omega^{2}}{\gamma}$

The terms on the right-hand side of Eq. (19) can all be expressed as a function of the geocentric latitude. Moreover, they can all be expressed in such a way that they can be expanded into converging binomial series. Firstly, the inverse of $\rho$ can be expressed as

$\frac{1}{\rho}=\frac{b}{a^{2}}\left[1-e^{2} \sin ^{2} \theta\right]^{\frac{3}{2}}\left[1-e^{2}\left(2-e^{2}\right) \sin ^{2} \theta\right]^{-\frac{3}{2}}$

The two terms between square brackets in Eq. (20) can be written as a binomial series

$$
\begin{aligned}
\frac{1}{\rho} & =\frac{b}{a^{2}}\left[\sum_{j=0}^{\infty}(-1)^{j}\left(\begin{array}{c}
\frac{3}{2} \\
j
\end{array}\right) e^{2 j} \sin ^{2 j} \theta\right] \\
& \times\left[\sum_{k=0}^{\infty}(-1)^{k}\left(\begin{array}{c}
-\frac{3}{2} \\
k
\end{array}\right) e^{2 k}\left(2-e^{2}\right)^{k} \sin ^{2 k} \theta\right]
\end{aligned}
$$

and these two binomial series can be combined using a Cauchy multiplication (a discrete version of convolution; e.g., Protter and Morrey 1964)

$$
\frac{1}{\rho}=\frac{b}{a^{2}} \sum_{j=0}^{\infty}(-1)^{j} e^{2 j} \sum_{k=0}^{j}\left(\begin{array}{l}
\frac{3}{2} \\
k
\end{array}\right)\left(\begin{array}{c}
-\frac{3}{2} \\
j-k
\end{array}\right)\left(2-e^{2}\right)^{j-k} \sin ^{2 j} \theta
$$

A power series for the inverse of $\nu$ can be found in a very similar way

$\frac{1}{\nu}=\frac{b}{a^{2}} \sum_{j=0}^{\infty}(-1)^{j} e^{2 j} \sum_{k=0}^{j}\left(\begin{array}{c}\frac{1}{2} \\ k\end{array}\right)\left(\begin{array}{c}-\frac{1}{2} \\ j-k\end{array}\right)\left(2-e^{2}\right)^{j-k} \sin ^{2 j} \theta$

The term $2 \omega^{2} / \gamma$ in Eq. (19) can also be expressed as a power series. For this purpose, the Somigliana-Pizzetti formula for reference gravity (Heiskanen and Moritz 1967 , p. 70) first needs to be expressed in spherical polar coordinates (Claessens 2006)

$$
\begin{array}{r}
\gamma=\gamma_{b}\left(1-e^{2} \sin ^{2} \theta\right)^{-\frac{1}{2}}\left[1-e^{2}\left(2-e^{2}\right) \sin ^{2} \theta\right]^{-\frac{1}{2}} \\
\times\left(1-e_{\gamma}^{2} \sin ^{2} \theta\right)
\end{array}
$$

where

$e_{\gamma}^{2}=1-\frac{\gamma_{a}}{\gamma_{b}}\left(1-e^{2}\right)^{\frac{3}{2}}$ and $\gamma_{a}$ and $\gamma_{b}$ are the magnitude of reference gravity at the equator and at the poles, respectively. The constant $e_{\gamma}^{2} \ll 1$, since $\gamma_{a} \approx \gamma_{b}$ and $e^{2} \ll 1$. The inverse of the reference gravity can therefore be written as the product of three fast-converging binomial series

$$
\begin{array}{r}
\frac{1}{\gamma}=\frac{1}{\gamma_{b}}\left[\sum_{i=0}^{\infty}(-1)^{i}\left(\begin{array}{c}
\frac{1}{2} \\
i
\end{array}\right) e^{2 i} \sin ^{2 i} \theta\right]\left[\sum_{j=0}^{\infty}(-1)^{j}\left(\begin{array}{c}
\frac{1}{2} \\
j
\end{array}\right)\right. \\
\left.\times e^{2 j}\left(2-e^{2}\right)^{j} \sin ^{2 j} \theta\right]\left[\sum_{k=0}^{\infty} e_{\gamma}^{2 k} \sin ^{2 k} \theta\right]
\end{array}
$$

The three binomial series can be combined using a Cauchy multiplication. Combining the first two series and subsequently combining it with the third, results in a single power series expression

$$
\begin{array}{r}
\frac{1}{\gamma}=\frac{1}{\gamma_{b}} \sum_{j=0}^{\infty} \sum_{k=0}^{j}(-1)^{k} e^{2 k} e_{\gamma}^{2(j-k)} \sum_{i=0}^{k}\left(\begin{array}{c}
\frac{1}{2} \\
i
\end{array}\right)\left(\begin{array}{c}
\frac{1}{2} \\
k-i
\end{array}\right) \\
\times\left(2-e^{2}\right)^{k-i} \sin ^{2 j} \theta
\end{array}
$$

Finally, adding the power series in Eqs. (22), (23) and (27) together and combining it with the power series of $\left(R / r_{\mathrm{e}}\right)^{(n+1)}$ gives

$$
\begin{aligned}
\left(\frac{R}{r_{\mathrm{e}}}\right)^{n+1} \frac{1}{\gamma} \frac{\partial \gamma}{\partial h}= & -\sum_{j=0}^{\infty} \sum_{k=0}^{j} \alpha_{n, j-k} \sum_{l=0}^{k}\left(\frac{b}{a^{2}}(-1)^{k}\right. \\
& \times\left(2-e^{2}\right)^{k-l} e^{2 k}\left[\left(\begin{array}{c}
\frac{3}{2} \\
l
\end{array}\right)\left(\begin{array}{c}
-\frac{3}{2} \\
k-l
\end{array}\right)\right. \\
+ & \left.\left(\begin{array}{c}
\frac{1}{2} \\
l
\end{array}\right)\left(\begin{array}{c}
-\frac{1}{2} \\
k-l
\end{array}\right)\right]+2 \frac{\omega^{2}}{\gamma_{b}}(-1)^{l} e^{2 l} e_{\gamma}^{2(k-l)} \\
& \left.\times \sum_{p=0}^{l}\left(\begin{array}{c}
\frac{1}{2} \\
p
\end{array}\right)\left(\begin{array}{c}
\frac{1}{2} \\
l-p
\end{array}\right)\left(2-e^{2}\right)^{l-p}\right) \sin ^{2 j} \theta
\end{aligned}
$$

This series can be used to obtain an expression for the second term of the fundamental equation of physical geodesy (Eq. (1)) in terms of the solid spherical harmonic coefficients of the disturbing potential. All dependence on latitude can be centred inside the spherical harmonic functions by application of Eq. (9)

$\frac{1}{\gamma} \frac{\partial \gamma}{\partial h} T=\sum_{n=0}^{\infty} \sum_{m=0}^{n} \bar{T}_{n m}^{R} \sum_{j=0}^{\infty} \sum_{i=-j}^{j} \varepsilon_{n m i j} \bar{Y}_{n+2 i, m}$

where

$$
\begin{aligned}
& \varepsilon_{n m i j}=-\bar{K}_{n m}^{2 i, 2 j} \sum_{k=0}^{j} \alpha_{n, j-k} \sum_{l=0}^{k}\left(\frac{b}{a^{2}}(-1)^{k}\left(2-e^{2}\right)^{k-l}\right. \\
& \times e^{2 k}\left[\left(\begin{array}{c}
\frac{3}{2} \\
l
\end{array}\right)\left(\begin{array}{c}
-\frac{3}{2} \\
k-l
\end{array}\right)+\left(\begin{array}{c}
\frac{1}{2} \\
l
\end{array}\right)\left(\begin{array}{c}
-\frac{1}{2} \\
k-l
\end{array}\right)\right]+2 \frac{\omega^{2}}{\gamma_{b}}(-1)^{l} \\
&\left.\times e^{2 l} e_{\gamma}^{2(k-l)} \sum_{p=0}^{l}\left(\begin{array}{c}
\frac{1}{2} \\
p
\end{array}\right)\left(\begin{array}{c}
\frac{1}{2} \\
l-p
\end{array}\right)\left(2-e^{2}\right)^{l-p}\right)(30)
\end{aligned}
$$

Equations (14) and (29) can be summed to obtain a formula for the gravity anomaly on the ellipsoid in 
terms of the solid spherical harmonic coefficients $\bar{T}_{n m}^{R}$, which after a rearrangement of summation order becomes

$$
\begin{array}{r}
\Delta g=\sum_{n=0}^{\infty} \sum_{m=0}^{n} \sum_{j=0}^{\infty} \sum_{i=-j-1}^{j+1}\left(\varepsilon_{n-2 i, m, i, j}+\frac{n-2 i+1}{R}\right. \\
\left.\times \beta_{n-2 i, m, i, j}+\frac{1}{R} \gamma_{n-2 i, m, i, j}\right) \bar{T}_{n-2 i, m}^{R} \bar{Y}_{n m}
\end{array}
$$

This expression can, due to the orthogonality of the spherical harmonic functions on the sphere, be directly compared to surface spherical harmonic coefficients of gravity anomalies on the ellipsoid (Eq. (3)). After a final rearrangement of summation order this gives

$\overline{\Delta g}_{n m}^{\mathrm{e}}=\sum_{i=-\infty}^{\infty} \lambda_{n m i}^{\mathrm{g}} \bar{T}_{n-2 i, m}^{R}$

where the weights $\lambda_{n m i}^{\mathrm{g}}$ can be computed from another infinite summation

$$
\begin{aligned}
\lambda_{n m i}^{\mathrm{g}}= & \sum_{j=|i|-1}^{\infty}\left(\varepsilon_{n-2 i, m, i, j}+\frac{n-2 i+1}{R}\right. \\
& \left.\times \beta_{n-2 i, m, i, j}+\frac{1}{R} \gamma_{n-2 i, m, i, j}\right)
\end{aligned}
$$

Equation (32) gives the spectral relationship between gravity anomalies and disturbing potential in ellipsoidal approximation. Though not as elegant as the simple one-to-one relation in SCR approximation, it is much more accurate. The simple one-to-one relation is nowadays no longer accurate enough for most practical applications (e.g., Barthelmes 2009). The accuracy of the ellipsoidal approximation derived here is tested numerically in the next section.

\section{A surface spherical harmonic model of gravity anomalies}

Using the coefficient transformation described in section 3.2, a surface spherical harmonic expansion of gravity anomalies with respect to the GRS80 ellipsoid (Moritz 2000) is computed from EGM2008 (Pavlis et al. 2012). For this purpose, the tide-free solid spherical harmonic coefficients of the gravitational potential provided by EGM2008 (up to maximum degree 2190) were transformed into unscaled solid spherical harmonic coefficients of the disturbing potential $\bar{T}_{n m}^{R}$ (e.g., Smith 1998), so that an expansion of the form of Eq. (2) is achieved. The zero- and first-degree coefficients were kept at zero. The reference gravity field employed in this transformation is the GRS80 reference gravity field.

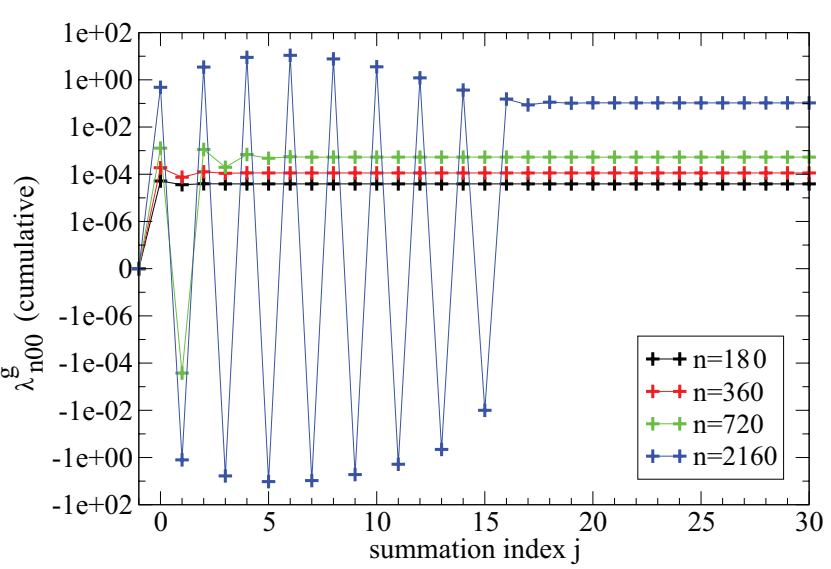

Fig. 1 Cumulative values of $\lambda_{n m i}^{\mathrm{g}}$ for various degrees $n$ with $m=0$ and $i=0$ in summation over $j$. Note: to optimally show the alternating nature of the series, the positive and negative parts of the vertical axis were independently scaled logarithmically (logarithm of absolute value was used for negative $\lambda_{n m i}^{\mathrm{g}}$ and axis was reversed), and combined into one graph by condensing all values $-1 e-08<\lambda_{n m i}^{\mathrm{g}}<1 e-08$ at zero

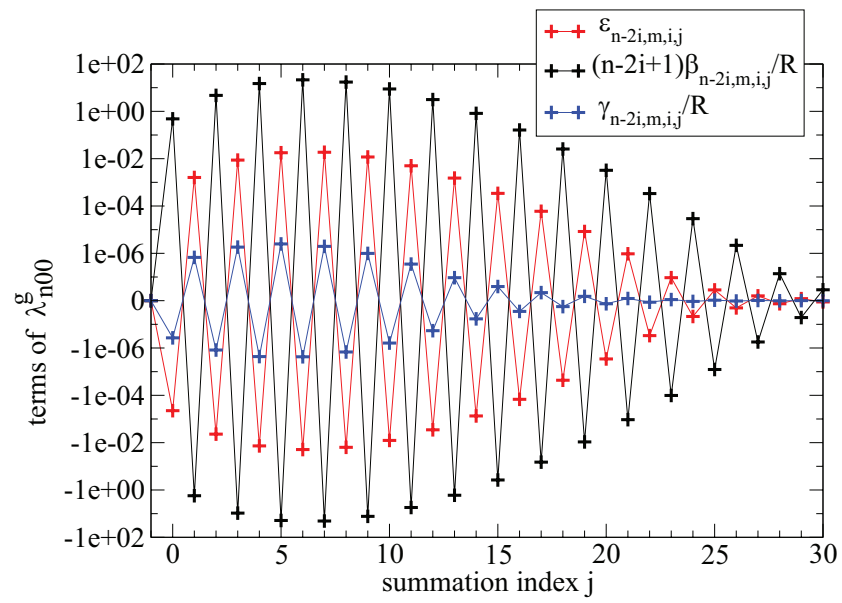

Fig. 2 Constituents of $\lambda_{n m i}^{\mathrm{g}}$ for $n=2160$ with $m=0$ and $i=0$. Note: to optimally show the alternating nature of the series, the positive and negative parts of the vertical axis were independently scaled logarithmically (logarithm of absolute value was used for negative $\lambda_{n m i}^{\mathrm{g}}$ and axis was reversed), and combined into one graph by condensing all values $-1 e-08<$ $\lambda_{n m i}^{\mathrm{g}}<1 e-08$ at zero 


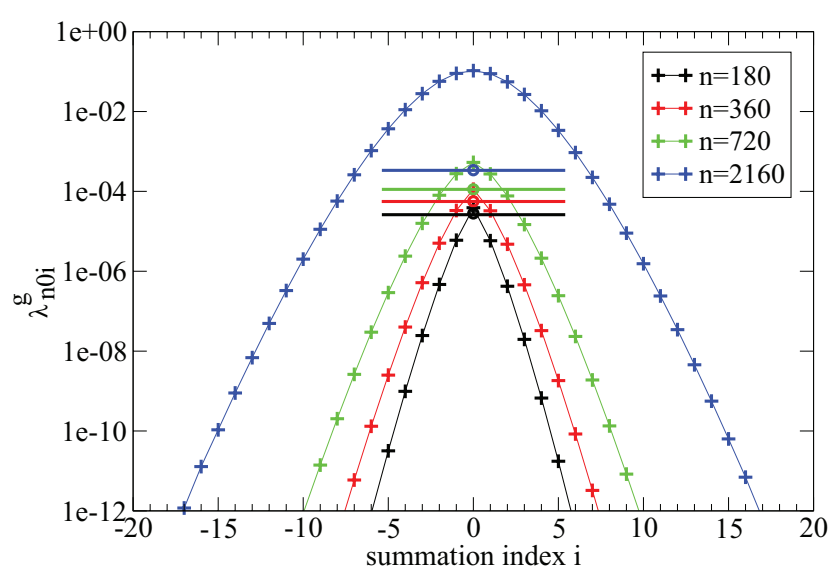

Fig. 3 Values of $\lambda_{n m i}^{\mathrm{g}}$ for various degrees $n$ with $m=0$; the circles with horizontal lines indicate the weights in SCR approximation $((n-1) / R)$

\subsection{Convergence}

It is important for an accurate transformation that the infinite summations in Eqs. (32) and (33) converge and are not truncated too early. Theoretically, the convergence rate of the series in Eq. (33) is slowest for high degrees, and dominated by the binomial expansion of $\left(R / r_{\mathrm{e}}\right)^{n+2}$ (Eq. (7)), which converges slowest of all expansions. Since this expansion is alternating, an upper limit for the truncation error $\varepsilon$ can be found using the d'Alembert ratio test (e.g., Stewart, 1995)

$\varepsilon<\frac{(n+1)^{j} e^{2 j}}{2^{j} j !}$

It follows from this that for $n=2160$ an acceptable truncation error of $e^{2}$ is achieved when 23 terms are taken into account. This is confirmed by numerical results shown in Figs. 1, 2 and 3. Only examples with $m=0$ are shown in these figures. The convergence rate of the series in Eq. (33) (Fig. 1) is similar for all values of $m$, while the convergence rate of the series in Eq. (32) (Fig. 3) is highest for larger values of $m$. Hence, overall convergence is slowest for $m=0$, making this a worst-case study.

Figure 1 shows the convergence of the series in Eq. (33) for various degrees $n$. As expected, it can be seen that the series is alternating, and that the oscillations become more volatile for higher degrees. The three constituents $\varepsilon_{n-2 i, m, i, j},(n-2 i+1) \beta_{n-2 i, m, i, j} / R$ and $\gamma_{n-2 i, m, i, j}$ $R$ that make up $\lambda_{n m i}(\Delta g, T)$ are shown in Fig. 2 for $n=2160, m=0$ and $i=0$. The term containing $\beta_{n-2 i, m, i, j}$ is the dominant term. To compute $\lambda_{n 00}^{\mathrm{g}}$ with

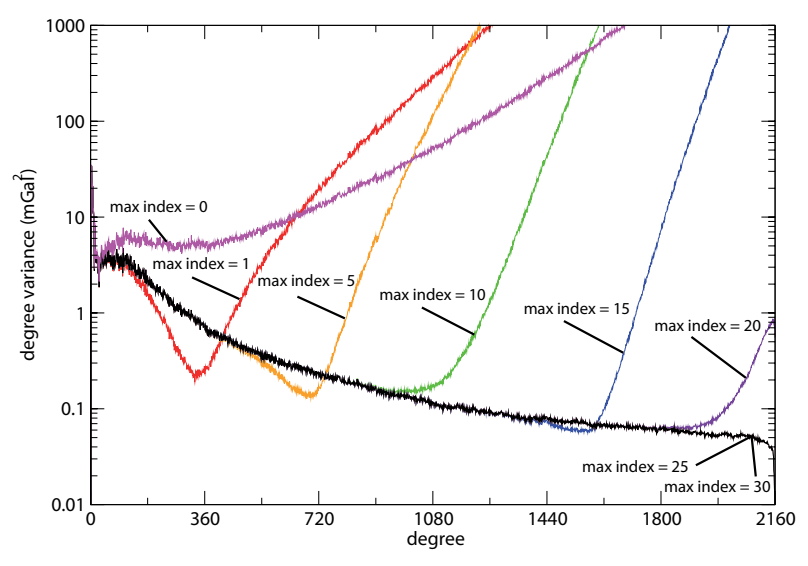

Fig. 4 Power spectrum of surface spherical harmonic series of gravity anomalies with various maximum summation indices, where $-i_{\min }=i_{\max }=j_{\max }$ equals the $\max$ index indicated

sufficient precision for all degrees up to $n=2160$, at least 25 terms need to be taken into account in the summation over $j$ (Eq. 33).

The convergence of the series in Eq. (32) is shown in Fig. 3. The values of $\lambda_{n m i}^{\mathrm{g}}$ decrease for indices $i$ further away from 0 , proving that the series converge fairly rapidly. However, simply using the value for $i=0$ only is clearly insufficient, especially for higher degrees, as the values for $|i|=1,2, \ldots$ are also significant. In SCR approximation, there is a one-to-one relation between spherical harmonic coefficients of disturbing potential and gravity anomaly (Eq. (4)), which comes down to having a value for $i=0$ only. The value for $\lambda_{n 00}^{\mathrm{g}}$ in SCR approximation is $(n-1) / R$ (Eq. (4)), and Fig. 3 shows that this is a clear underestimation, especially for high degrees.

To test the effect of series truncation on the solution, the infinite summations were truncated at various values of $i$ and $j$. The spherical harmonic power spectra of the gravity anomaly solutions are plotted in Fig. 4 for various maximum truncation indices, which were set equal for $i$ and $j$, thus $-i_{\min }=i_{\max }=j_{\max }$. As expected, the number of terms in the summation must be increased to perform the coefficient transformation with high precision up to ever higher degree and order. The effect of truncation at too low a value on the power spectrum is dramatic, which is due to the volatile oscillations in the summation over $j$ (cf. Fig. 1). Maximum truncation indices of at least $-i_{\min }=i_{\max }=j_{\max }=25$ are required to obtain a realistic spectrum up to degree and order 2160. The spectra for summations up to 25 and 30 are indistinguishable in Fig. 4. 


\subsection{Computation and validation}

The disturbing potential coefficients derived from EGM2008 were transformed into gravity anomaly coefficients using Eq. (32). The infinite summation in Eq. (32) was truncated from $i_{\min }=-30$ to $i_{\max }=30$, and in Eq. (33) to $j_{\max }=30$. All coefficients $\bar{T}_{n m}^{R}$ of negative degree or of degrees beyond the maximum of 2190 were set to zero. This resulted in a surface spherical harmonic expansion of gravity anomalies up to degree and order 2250. Note that the coefficients beyond degree $\sim 2130$ are not complete, since coefficients beyond degree 2190 are needed to compute them correctly, and additionally, EGM2008 coefficients are incomplete beyond degree 2159. Yet all surface spherical harmonic coefficients to degree and order 2250 are retained to preserve the band-limited content of the expansion. Note also that this expansion contains zero- and first-degree terms that are non-zero. A discussion on the zero- and first degree surface spherical harmonic coefficients of gravity anomalies is provided in Heck and Seitz (2003).

The surface spherical harmonic expansion of gravity anomalies is validated by a global spherical harmonic synthesis. Point values of gravity anomalies on the GRS80 ellipsoid are computed from the computed gravity anomaly expansion on a global $10^{\prime} \times 10^{\prime}$ grid. These are compared to point values of linearly approximated gravity anomalies computed from EGM2008 using the harmonic synth software provided by the EGM2008 development team (Holmes and Pavlis 2006). In harmonic synth, the so-called linearly approximated gravity anomalies are computed according to Eq. (1), or more specifically according to Eq. (6.33) in Claessens (2006). The gravity anomalies were computed directly from EGM2008 coefficients, i.e. the spherical harmonic coefficients of the gravitational potential as provided by EGM2008 were used as input in harmonic synth. Therefore, the validation includes the transformation from EGM2008 coefficients to unscaled solid spherical harmonic coefficients of the disturbing potential, as well as the subsequent transformation to surface spherical harmonic coefficients of the gravity anomalies.

The differences between both grids are shown in Fig. 5 and Table 1. It can be seen that with $i_{\max }=$ 30 , the agreement between both grids of gravity anomalies is worst near the equator, but $<1 \mu \mathrm{Gal}$ everywhere on Earth and the global RMS of differences is just $0.08 \mu \mathrm{Gal}$. The pattern of poorer precision near the equator vanishes completely when $i_{\max }$ is increased to $40\left(i_{\min }=-40, j_{\max }=40\right)$ and only numerical rounding errors remain in the statistics (Table 1). This proves the very high precision of the coefficient transformation
Table 1 Global statistics of the comparison of gravity anomalies from a surface spherical harmonic expansion in SCR approximation, in Heck and Seitz (2003) approximation ('HS'), and in ellipsoidal approximation with $i_{\max }=30$ ('ell(i=30)') and $i_{\max }=40$ ('ell(i=40)') against gravity anomalies directly synthesised from EGM2008 (units in mGal)

\begin{tabular}{lrrrr}
\hline & SCR & HS & ell $(\mathrm{i}=30)$ & ell(i=40) \\
\hline minimum & -192.26 & -183.16 & $-5.11 \cdot 10^{-4}$ & $-1.53 \cdot 10^{-5}$ \\
maximum & 319.67 & 245.18 & $6.10 \cdot 10^{-4}$ & $1.53 \cdot 10^{-5}$ \\
mean & 0.0459 & 0.0525 & $2.53 \cdot 10^{-8}$ & 0.00 \\
RMS & 10.99 & 6.09 & $8.31 \cdot 10^{-6}$ & $2.86 \cdot 10^{-8}$ \\
\hline
\end{tabular}

derived in section 3.2 to degree and order 2190, sufficient for any practical application.

Table 1 also shows the global statistics for gravity anomalies obtained using Eq. (51) in Heck and Seitz (2003). The formula provided by Heck and Seitz (2003) is essentially an approximation of the rigorous formulas derived in Section 3 (Eq. 32). Heck and Seitz (2003) use this formula as an intermediate result in the derivation of ellipsoidal corrections to Stokes's formula, and acknowledge that the approximation is poor in the computation of high degree surface spherical harmonic coefficients of gravity anomalies. This is confirmed and quantified by the results in Table 1 . The main reasons for the poor performance in this comparison is that the Heck and Seitz (2003) formula only takes into account terms of $i=-1,0,1$, which as shown by Figs. (1-3) is insufficient for high degrees. Since gravity anomalies possess high power in the high degrees, the statistics are poor as a result. However, the Heck and Seitz (2003) formula does provide a great improvement upon the SCR approximation for low degree coefficients, as can be seen in Fig. 6 .

Figure 6 shows the degree variance spectra of the surface spherical harmonic expansion of gravity anomalies with respect to the GRS80 ellipsoid in 1) SCR approximation, 2) Heck and Seitz (2003) approximation, and 3) ellipsoidal approximation (rigorous solution). For the SCR approximation, the reference radius $R$ in the factor $(n-1) / R$ was set equal to the mean Earth radius $(6371005 \mathrm{~m})$. Note that the coefficients $T_{n m}^{R}$ were not rescaled, so the reference radius $R$ in the term $(R / r)^{n+1}$ in Eq. (2) was left at the EGM2008 value of $6378136.3 \mathrm{~m}$.

It can be seen that the SCR approximation underestimates the power in the spectrum by up to almost one order of magnitude for the highest degrees. This is because the SCR approximation essentially represents the spectrum on the ellipsoid's bounding sphere, where the gravity signal is strongly attenuated (away from the equator). This is consistent with the result shown in Fig. 3 which suggests that the SCR factor of $(n-1) / R$ 


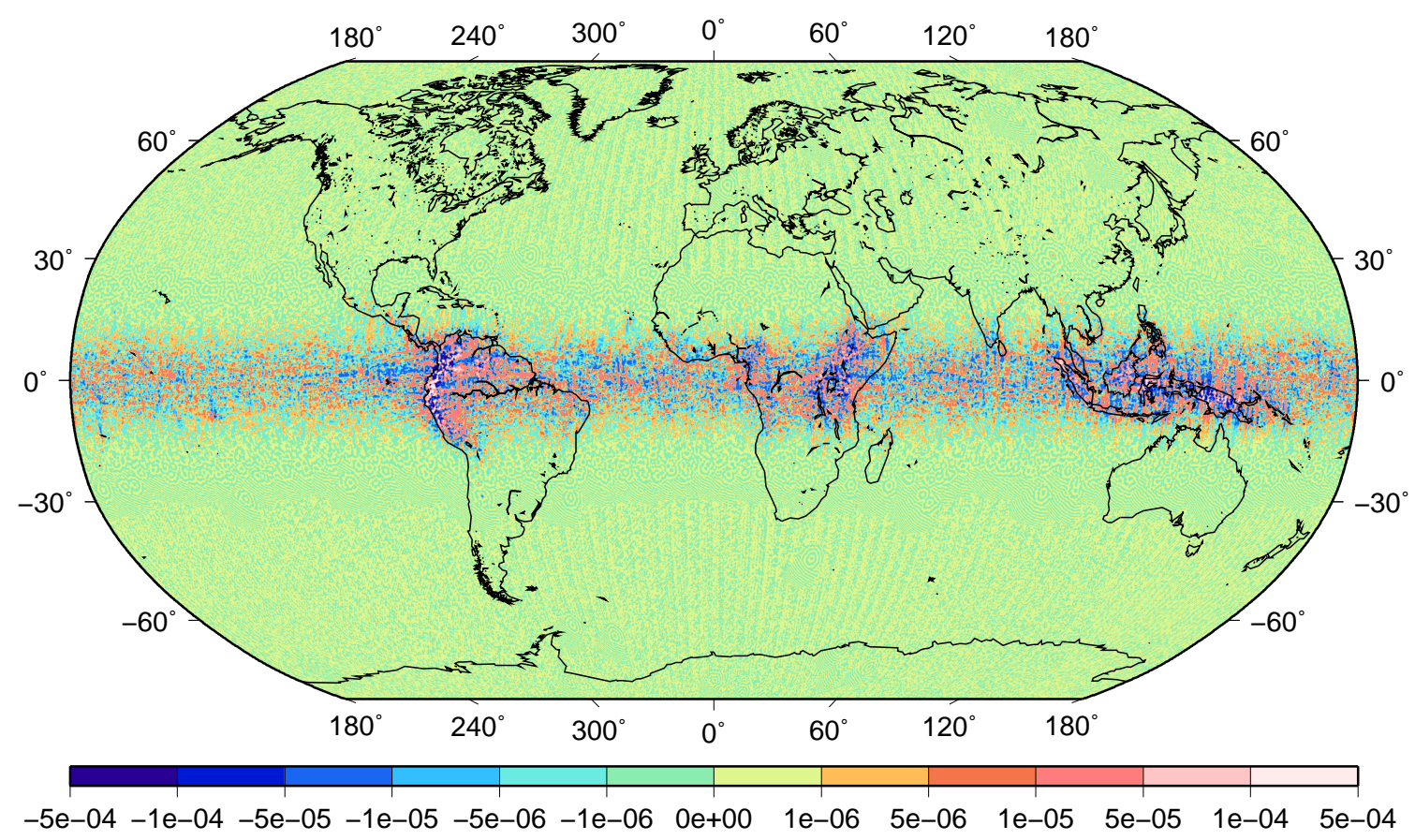

Fig. 5 Differences between gravity anomalies computed from surface harmonic expansion of gravity anomalies and from EGM2008 $\left(i_{\max }=30\right.$ used; units in mGal; Robinson projection)

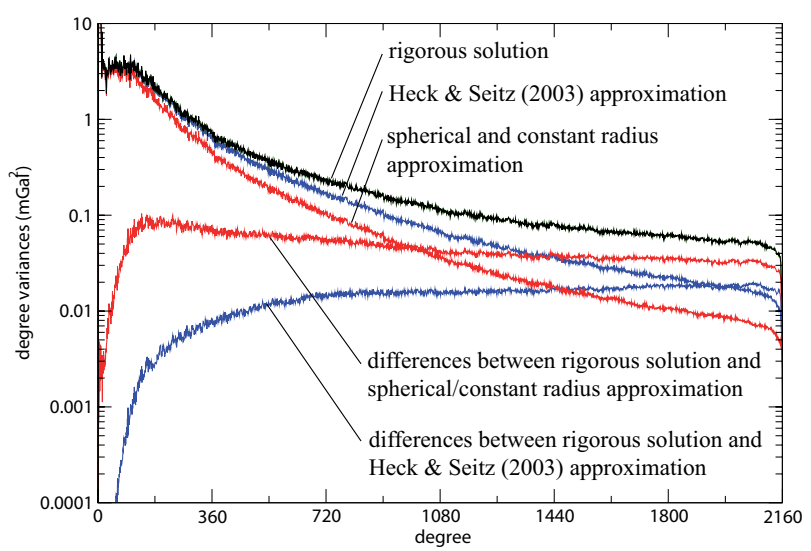

Fig. 6 Power spectra of surface spherical harmonic series of gravity anomalies in SCR approximation, in Heck and Seitz (2003) approximation, and in ellipsoidal approximation (black line), and of the differences between the series

is too small. It should thus never be assumed that the spectrum in SCR approximation is representative of the spectrum on the ellipsoid or the Earth's topography, particularly not for high spherical harmonic degrees.

Figure 7 shows that the SCR approximation has similar power to the rigorous ellipsoidal approximation when the spectrum is evaluated on a sphere with a radius of $\sim 6375000 \mathrm{~m}$. The differences between degree

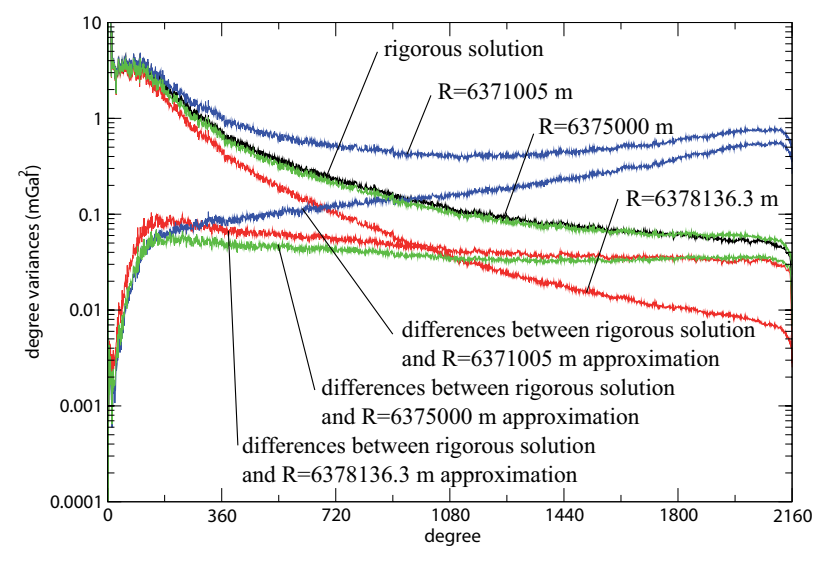

Fig. 7 Comparison of power spectra of surface spherical harmonic series of gravity anomalies in SCR approximation with varying reference radii to power spectrum of rigorous ellipsoidal solution

variances in SCR and ellipsoidal approximation are generally smallest with $R \approx 6375000$. They reach a maximum at degree $\sim 150$ and taper off very slowly. The Heck and Seitz (2003) approximation is much more accurate for low degree coefficients, but also underestimates the power in the high degrees (Fig. 6). 


\section{Comparison to Hotine-Jekeli transformation}

The transformation presented here (Eq. (32)) has some similarities with the well-known transformation between spherical harmonic coefficients and ellipsoidal harmonic coefficients, known in geodesy as the Hotine-Jekeli transformation (Jekeli 1988, Sebera et al. 2012). Both transformations present a relation between spherical and ellipsoidal spectra, and in both the relation is given as a weighted summation over coefficients of equal order but a range of degrees. The main difference is that the Hotine-Jekeli transformation uses ellipsoidal harmonics (using ellipsoidal coordinates) whereas the transformation presented here uses surface spherical harmonics on the ellipsoid (using spherical coordinates).

The Hotine-Jekeli transformation as described in Jekeli (1988) is a transformation between harmonic coefficients of one and the same quantity. Gleason (1988) shows how the method can be applied for the transformation of spherical harmonic coefficients of the disturbing potential or gravitational potential to ellipsoidal harmonic coefficients of gravity anomalies and vice versa. The forward transformation (i.e. from disturbing or gravitational potential to gravity anomalies) is performed using Eqs. (2.4) and (2.8) in Gleason (1988) which in the current notation read

$\bar{g}_{n m}^{R}=\frac{n-1}{R} \bar{T}_{n m}^{R}$

and

$\bar{g}_{n m}^{\mathrm{e}}=\bar{S}_{n|m|}\left(\frac{b}{E}\right) \sum_{i=0}^{w} \lambda_{n m i} \bar{g}_{n-2 i, m}^{R}$

where $R \bar{g}_{n m}^{\mathrm{e}}$ are the real ellipsoidal harmonic coefficients of the harmonic quanitity $r \widetilde{\Delta g}, \bar{S}_{n m}\left(\frac{b}{E}\right)$ is Jekeli's renormalised Legendre function of the second kind, $\lambda_{n m i}$ are weights that are computed recursively (Gleason 1988, Eq. 1.18), and

$w=\operatorname{int}\left(\frac{n-m}{2}\right)$

Equation (35) follows from the definition of gravity anomalies in spherical approximation (Eq. (5)). This means that the harmonic coefficients of gravity anomalies computed using the Hotine-Jekeli transformation as per Gleason (1988) (Eqs. (35-37)) are harmonic coefficients of gravity anomalies in spherical approximation (which should not be confused with the fact that they are ellipsoidal harmonic coefficients). The transformation can therefore be compared to that derived in section 3.1 (Eq. (12)). However, the Hotine-Jekeli transformation cannot directly be applied to provide harmonic coefficients of gravity anomalies in ellipsoidal approximation (Eq. (1)), so it is not directly comparable to the transformation derived in section 3.2 (Eq.
(32)). In other words, while the transformation presented here (Eq. (32)) yields an ellipsoidal spectrum of gravity anomalies in ellipsoidal approximation, it is not obvious how the same could be achieved with the Hotine-Jekeli transformation.

The differences between harmonic coefficients of gravity anomalies in spherical and in ellipsoidal approximation are significant, especially in the higher degrees. The Hotine-Jekeli transformation is regularly applied for the reverse of the case discussed here, i.e., computing spherical harmonic coefficients of the disturbing or gravitational potential from gravity anomalies (e.g. Lemoine et al. 1998, Holmes and Pavlis 2007, Pavlis et al. 2012). In that case, ellipsoidal corrections to order $e^{2}$ are usually applied to gravity anomalies in the space domain to accommodate for these differences (e.g., Rapp and Pavlis 1990). In the transformation presented here (Eq. (32)), ellipsoidal corrections in the space domain are not required. It provides a one-step, rigorous transformation between surface spherical harmonic coefficients of gravity anomalies in ellipsoidal approximation and solid spherical harmonic coefficients of the disturbing potential, fully in the spectral domain.

Apart from the above, one obvious difference between the transformation presented here and the HotineJekeli transformation is the selection of coordinates. In the Hotine-Jekeli transformation, two types of coordinates are used: geocentric spherical polar coordinates (using geocentric co-latitude) for the sphere and ellipsoidal coordinates (using reduced co-latitude) for the ellipsoid. In our method, only geocentric spherical polar coordinates are used, i.e. data on both the sphere and the ellipsoid is parametrised by geocentric co-latitude and longitude. A minor advantage of the use of only geocentric coordinates is that all global gravity models are currently provided in solid spherical harmonic coefficients which use geocentric coordinates and transformation to another coordinate system is not required. Gruber and Abrykosov (2014) further describe the inconvenience of the need to use reduced co-latitude in the Hotine-Jekeli transformation and provide a method to use the Hotine-Jekeli transformation when data on the ellipsoid are provided in terms of geocentric or geodetic latitude.

In terms of the rate of series convergence, the HotineJekeli transformation and our method are similar. Both methods require a weighted summation over coefficients of equal order. A difference is the range of degrees required. Upon comparison of Eq. (36) with Eq. (32), it can be seen that the Hotine-Jekeli transformation only requires spherical harmonic coefficients of lower degree, while our transformation requires coefficients of both lower and higher degree. It can also be seen 
that the Hotine-Jekeli transformation is a finite series, whereas our transformation is an infinite series. However, in practice both series are not applied in full but truncated, and both series converge at a similar rate. In addition, if the function on the sphere is band-limited, both methods lead to an ellipsoidal spectrum with infinite bandwidth, but with coefficients that decrease rapidly beyond the maximum degree of the function on the sphere.

The calculation of Jekeli's renormalised Legendre function of the second kind involves an infinite series that is to be truncated when sufficient precision is obtained (Jekeli 1988, Eq. (33)). Sebera et al. (2012) provide formulas that accelerate the convergence of the renormalised Legendre function of the second kind. This infinite series can be compared to the infinite series in our calculation of $\lambda_{n m i}^{\mathrm{g}}$ (Eq. (33)). The number of terms that needs to be taken into account is of the same order in both methods, and computation time is not restrictive for practical application of either method.

\section{Applications}

Several applications of a surface harmonic expansion of gravity anomalies are mentioned in the introduction. Two of these are presented in more detail here.

6.1 Computation of ellipsoidal corrections to Stokes's formula

Many authors have derived corrections to the spherical approximation in Stokes's formula for geoid determination (e.g., Fei and Sideris 2000, Heck and Seitz 2003, Sjöberg 2003). It has long been recognised that such ellipsoidal corrections are predominantly of a longwavelength nature (Lelgemann 1970, Rapp 1981). Huang et al. (2003) show that the contribution of spherical harmonic coefficients beyond degree 20 only contributes $10 \%$ of the total ellipsoidal corrections. It is therefore very efficient to model ellipsoidal corrections using a spherical harmonic expansion, and compute ellipsoidal corrections from a global gravity model.

Heck and Seitz (2003) and Sjöberg (2003) have derived specific approximate formulas to compute a surface harmonic expansion of ellipsoidal corrections. However, computing ellipsoidal corrections in an exact manner is very straightforward when a surface harmonic expansion of gravity anomalies is available. This follows from the spectral form of Stokes's formula.

Stokes's formula only holds for a spherical reference surface, but is typically applied to gravity anomalies on or close to the reference ellipsoid. Applying Stokes's formula over gravity anomalies on the ellipsoid therefore gives height anomalies (quasi-geoid heights) that are in error, $\tilde{\zeta}$. While Stokes's formula is usually evaluated in the space domain using numerical integration (e.g., Hirt et al. 2011), it can also be expressed in spectral form (cf. Heiskanen and Moritz, 1967, p.97)

$\tilde{\zeta}=\frac{1}{\gamma} \sum_{n=2}^{\infty} \sum_{m=-n}^{n} \frac{R_{s}}{n-1} \overline{\Delta g}_{n m}^{\mathrm{e}} \bar{Y}_{n m}$

where $R_{s}$ is the Stokesian reference radius. The height anomaly without spherical approximation, $\zeta$, can be computed from a global gravity model (Eq. (2)) and Bruns's equation

$\zeta=\frac{1}{\gamma} \sum_{n=2}^{\infty} \sum_{m=-n}^{n}\left(\frac{R}{r_{\mathrm{e}}}\right)^{n+1} \bar{T}_{n m}^{R} \bar{Y}_{n m}$

assuming that all appropriate ellipsoidal corrections were applied in the creation of the global gravity model. Ellipsoidal corrections to the unmodified Stokes kernel, $\delta \zeta$, can simply be found from the difference between Eqs. (38) and (39)

$\delta \zeta=\zeta-\tilde{\zeta}$

Ellipsoidal corrections to Stokes's formula computed in this manner are shown in Fig. 8. Here, the EGM2008 global gravity model and the GRS80 reference ellipsoid were used, and the Stokesian reference radius was set equal to the semi-major axis of the GRS80 ellipsoid. The surface spherical harmonic coefficients of gravity anomalies were computed via the transformation derived in Section 3, where the infinite summation over $i$ was restricted from $i_{\min }=-30$ to $i_{\max }=30$.

Figure 9 shows the differences between these ellipsoidal corrections and those computed using Eq. (59) in Heck and Seitz (2003). The RMS of the differences is $4.2 \mathrm{~mm}$, commensurate with Heck and Seitz's (2003) statement that their formula is a solution to order $e^{3}$. The spatial pattern of the differences is similar to the pattern of the ellipsoidal corrections themselves, and they are primarily of a long-wavelength nature. Shortwavelength differences are also visible away from the equator, where the ellipsoidal radius is furthest apart from the Stokesian reference radius selected here (the semi-major axis of the reference ellipsoid). This selection of the Stokesian reference radius was made, because the Heck and Seitz (2003) formula only applies to this choice. The method presented here, on the other hand, is not only more accurate but also more general, as it can easily use any Stokesian reference radius. Claessens (2006) has shown that it is best to choose the Stokesian reference radius equal to the ellipsoidal radius of the computation point. 


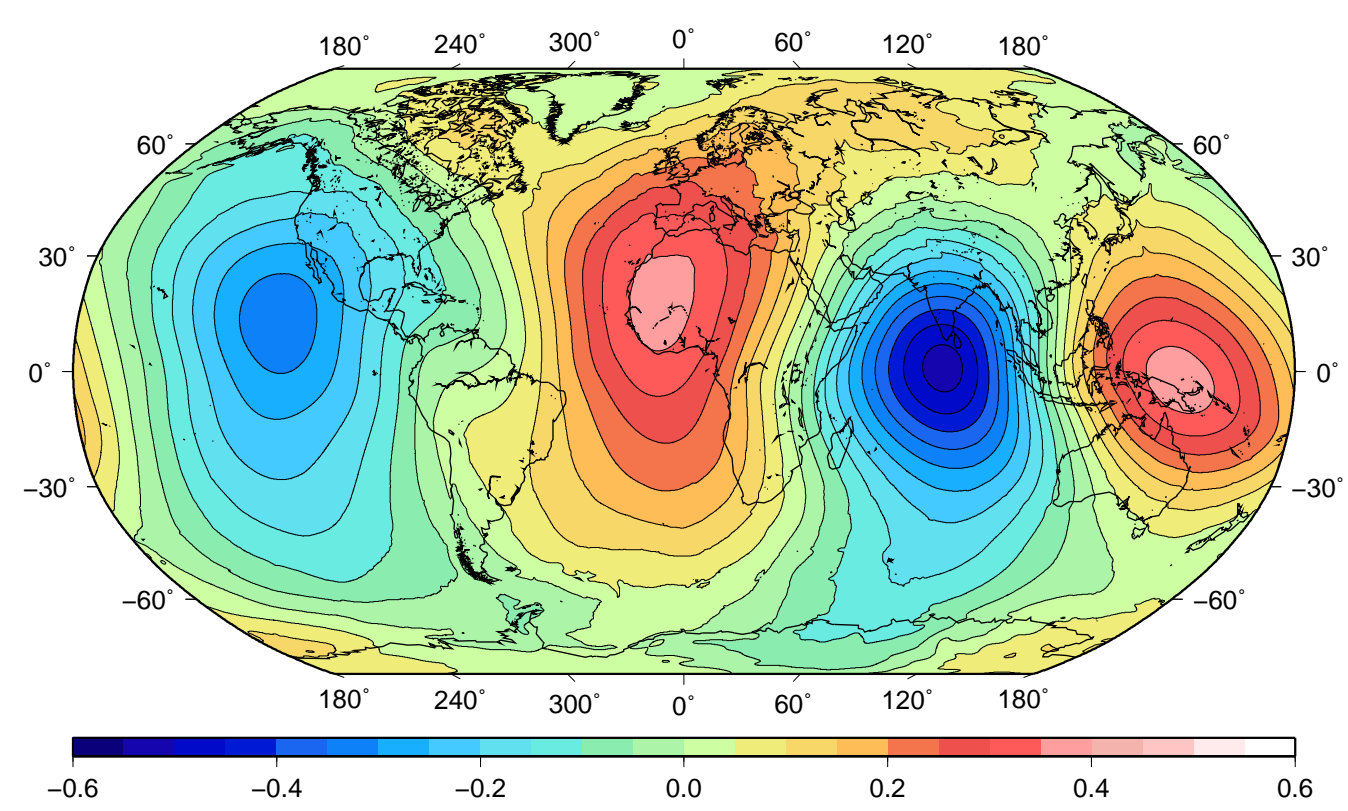

Fig. 8 Ellipsoidal corrections to height anomalies from Stokes's formula (units in m; Robinson projection)

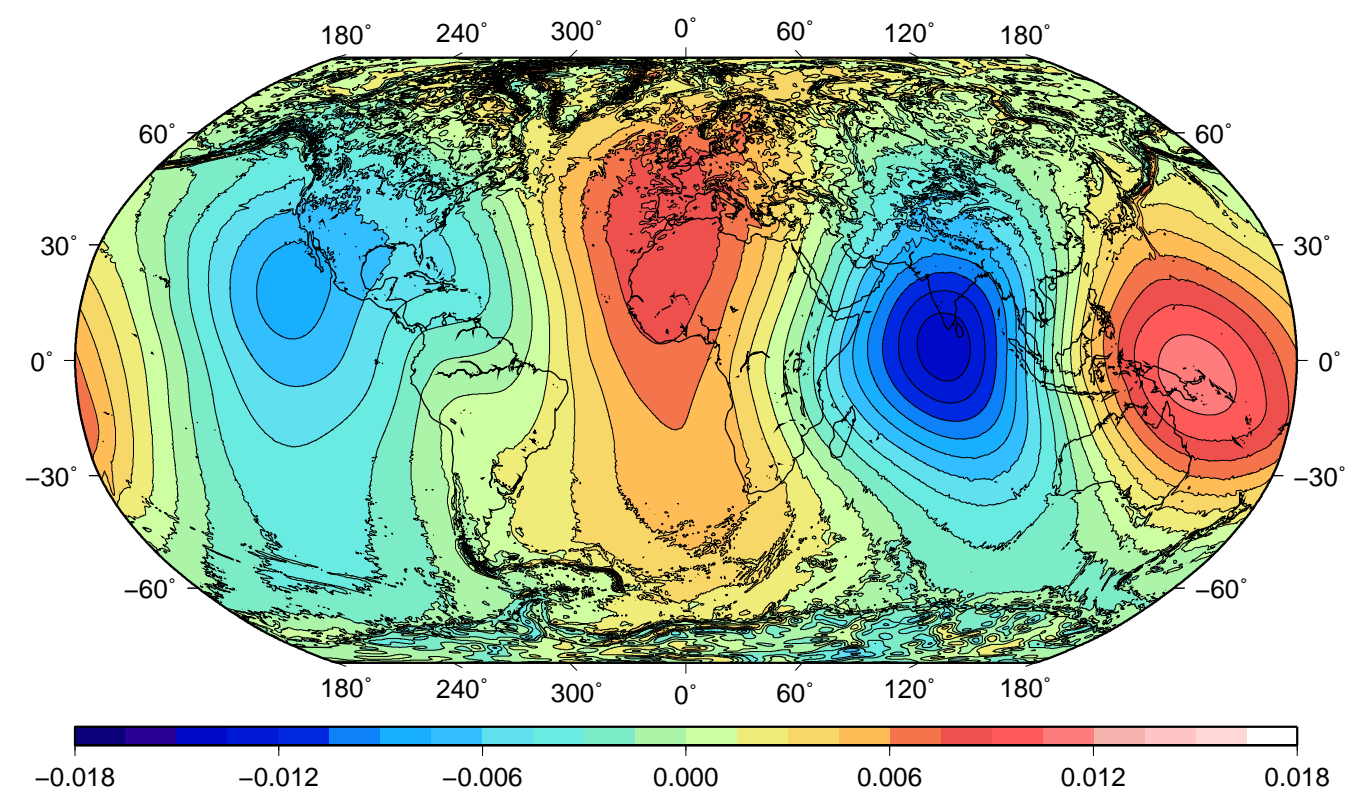

Fig. 9 Differences between ellipsoidal corrections computed using Eqs. (38-40) and ellipsoidal corrections computed using Eq. (59) in Heck and Seitz (2003) (units in m; Robinson projection)

The method for computation of ellipsoidal corrections can be extended to the commonly applied combined geoid determination where a global gravity model is used to model the long wavelengths of the geoid. It can also be extended to be used with modified Stokes kernels, but this is outside the scope of this paper.
6.2 Computation of ellipsoidal area means of gravity anomalies

Area means of gravity anomalies computed from global gravity models are routinely used in gravimetric geoid computation. In SCR approximation, area means of gravity anomalies can directly be computed from a global gravity model using recurrence relations for integrals of associated Legendre functions (Paul 1978). However, gravity anomalies in SCR approximation are of insuffi- 

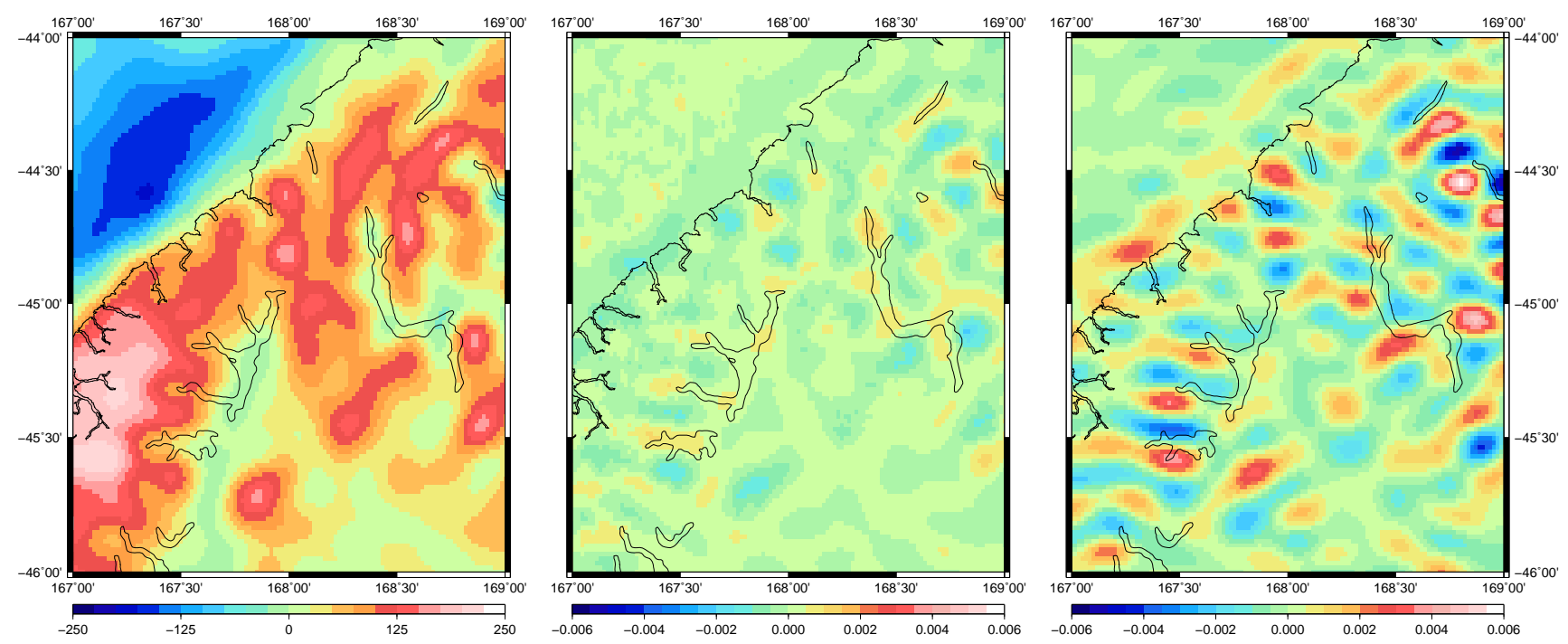

Fig. 10 Area means of gravity anomalies computed using the surface harmonic method in a test area on New Zealand's South Island (left), differences between the surface harmonic method and the fine-grid method (centre), and differences between the surface harmonic method and the three-grid method (right) (units all in mGal; Mercator projection)

cient precision to be used in modern geoid determination (Hipkin 2004).

It is fairly straightforward to compute point values of gravity anomalies from a global gravity model in ellipsoidal approximation (Hipkin 2004, Claessens 2006), but the computation of area means of gravity anomalies in ellipsoidal approximation from a global gravity model is more complicated. This is because in ellipsoidal approximation there is no one-to-one relation between solid spherical harmonic coefficients of the disturbing potential and surface spherical harmonic coefficients of gravity anomalies, which complicates the application of the recurrence relations of Paul (1978). For this reason, the harmonic synth software for spherical harmonic synthesis can compute area means of gravity anomalies in SCR approximation, but not in ellipsoidal approximation.

While it is complicated to compute area means of gravity anomalies directly from a global gravity model, it is straightforward to compute these area means from a surface spherical harmonic expansion of gravity anomalies, because in this case the recurrence relations of Paul (1978) can be used. Thus, the rigorous computation of area means of gravity anomalies in ellipsoidal approximation can be performed using a two-step procedure: first the spherical harmonic coefficient transformation described in section 3.2 , followed by synthesis of area means. This is here denoted the surface harmonic method.

To validate this procedure numerically, area means of gravity anomalies are computed over $1^{\prime} \times 1^{\prime}$ cells in a $2^{\circ} \times 2^{\circ}$ test area (a mountainous part of New
Zealand's South Island; Fig. 10, left). These are compared to area means obtained using two other methods, here denoted the fine-grid method and the threegrid method (Hirt and Claessens 2011). In the fine-grid method, point values of gravity anomalies in ellipsoidal approximation are synthesised on a very dense $3^{\prime \prime} \times 3^{\prime \prime}$ grid, after which the blockmean of these values is computed for each $1^{\prime} \times 1^{\prime}$ cell. In the three-grid method, area means of gravity anomalies in SCR approximation are synthesised first, after which an ellipsoidal correction is added. This ellipsoidal correction is the difference between point values of gravity anomalies in ellipsoidal and in SCR approximation, evaluated in the centre of each cell. Both the fine-grid and three-grid methods are approximations. The precision of the fine-grid method can be increased by creating a denser grid, at the expense of additional computation time. The finegrid method is significantly slower than the other two methods, and therefore not suited to large computation areas.

Of the three methods, the surface harmonic method and the fine-grid method show the closest agreement. This can be seen from the centre and right figures in Fig. 10. The centre figure shows that the differences between the surface harmonics method and the finegrid method are less than $3 \mu \mathrm{Gal}$ anywhere in the test area. The three-grid method appears to be the least precise of the three, but the level of agreement between all three methods shows that all are of sufficient precision for practical application at current. The surface harmonic method is theoretically the most rigorous, and also the most efficient and easiest to implement if a sur- 
face spherical harmonic expansion of gravity anomalies is readily available.

\section{Conclusions}

A surface spherical harmonic expansion of gravity anomalies with respect to a reference ellipsoid can be computed directly from a solid spherical harmonic expansion of the disturbing potential (or a global gravity model) through a coefficient transformation. Since in ellipsoidal approximation there is no one-to-one relation between the coefficients of both expansions, the coefficient transformation utilises a summation over coefficients of equal order $m$ but different degree $n$. The transformation has been validated by comparison of synthesised grids of gravity anomalies from both expansions, which agree with a global RMS precision of well below 1 nanoGal. The spectra of gravity anomalies under different levels of approximation show that approximations to order $e^{2}$ or $e^{3}$ are insufficient for the generation of surface spherical harmonic coefficients with respect to an ellipsoid. The Hotine-Jekeli transformation between solid spherical and ellipsoidal harmonic coefficients (Jekeli 1988) using the scheme of Gleason (1988) is also incapable of producing an ellipsoidal spectrum of gravity anomalies in ellipsoidal approximation, because it relies on a spherical approximation of gravity anomalies in the spectral domain. Our new transformation overcomes this limitation of the Hotine-Jekeli transformation. Among other applications, a surface spherical harmonic expansion of gravity anomalies in ellipsoidal approximation can efficiently and successfully be used to compute ellipsoidal corrections to gravimetric geoid computation and to compute area means of gravity anomalies.

Acknowledgements We would like to thank the Australian Research Council for funding this research through discovery project grants DP120102441 and DP0663020. Thanks are also extended to the reviewers and editors of this manuscript.

\section{References}

1. Albertella A, Sacerdote F, Sansò F (1993) Geodetic calculus with block-averages observations on the sphere. Surv in Geophys, 14:395-402, doi:10.1007/BF006905567

2. Barthelmes F (2009) Definition of functionals of the geopotential and their calculation from spherical harmonic models. Technical Report S09/02, Helmholtz-Zentrum Potsdam, Deutsches GeoForschungsZentrum, Germany

3. Buchdahl HA, Buchdahl NP, Stiles PJ (1977) On a relation between spherical and spheroidal harmonics. J. Phys. A: Math. Gen., 10:1833-1836
4. Claessens SJ (2005) New relations among associated Legendre functions and spherical harmonics. J Geod, 79:398406, doi:10.1007/s00190-005-0483-9

5. Claessens SJ (2006) Solutions to ellipsoidal boundary value problems for gravity field modelling. Dissertation, Curtin University of Technology, Perth, Australia

6. Claessens SJ, Featherstone WE (2008) The Meissl scheme for the geodetic ellipsoid. J Geod, 82:513-522, doi: $10.1007 / \mathrm{s} 00190-007-0200-\mathrm{y}$

7. Claessens SJ, Hirt C, Amos MJ, Featherstone WE, Kirby JF (2011) The NZGeoid09 model of New Zealand. Surv Rev, 43:2-15, doi: 10.1179/003962610X12747001420780

8. Claessens SJ, Hirt C (2013) Ellipsoidal topographic potential: New solutions for spectral forward gravity modeling of topography with respect to a reference ellipsoid. J Geophys Res Solid Earth, 118:5991-6002, doi:10.1002/2013JB010457.

9. Colombo OL (1981) Numerical methods for harmonic analysis on the sphere. Report 310, Dept of Geod Sci and Surv, Ohio State University, Columbus

10. Cruz JY (1986) Ellipsoidal corrections to potential coefficients obtained from gravity anomaly data on the ellipsoid. Report 371, Dept of Geod Sci and Surv, Ohio State University, Columbus

11. Featherstone WE, Evans JD, Oliver JG (1998) A Meisslmodified Vaníček and Kleusberg kernel to reduce the truncation error in gravimetric geoid computation. J Geod, 72:154-160, doi:10.1007/s001900050157

12. Featherstone WE, Kirby JF, Hirt C, Filmer MS, Claessens SJ, Brown N, Hu G, Johnston GM (2011) The AUSGeoid09 model of the Australian Height Datum. J Geod, 85:133-150, doi:10.1007/s00190-010-0422-2

13. Fei ZL, Sideris MG (2000) A new method for computing the ellipsoidal correction for Stokes's formula. J Geod, 74:223-231, doi:10.1007/s001900050280 (erratum in 74:671, doi:10/1007/s001900000131)

14. Gleason DM (1988) Comparing ellipsoidal corrections to the transformation between the geopotential's spherical and ellipsoidal spectrums. manu geod, 13:114-129

15. Grafarend EW, Engels J (1994) The convergent series expansion of the gravity field of a starshaped body. manu geod, 19:18-30

16. Gruber C, Abrykosov O (2014) High resolution spherical and ellipsoidal harmonic expansions by Fast Fourier Transform. Stud Geophys et Geod, 58:595-608, doi:10.1007/s11200-013-0578-3

17. Heck B, Seitz K (2003) Solutions of the linearized geodetic boundary value problem for an ellipsoidal boundary to order $e^{3}$. J Geod, 77:182-192, doi:10.1007/s00190-002-0309-

18. Heiskanen W, Moritz H (1967) Physical Geodesy. WH Freeman \& Co, San Fransisco, USA

19. Hipkin RG (2004) Ellipsoidal geoid computation. J Geod, 78:167-179, doi:10.1007/s00190-004-0389-y

20. Hirt C, Claessens SJ (2011) Ellipsoidal area mean gravity anomalies - precise computation of gravity anomaly reference fields for remove-compute-restore geoid determination. Stud Geophys et Geod, 55: 589-607, doi: 10.1007/s11200010-0070-2

21. Hirt C, Featherstone WE, Claessens SJ (2011) On the accurate numerical evaluation of geodetic convolution integrals. J Geod, 85:519-538, doi:10.1007/s00190-011-0451-5

22. Holmes SA, Pavlis NK (2006) A Fortran program for very-high-degree harmonic synthesis (version 05/01/2006), http://earthinfo.nga.mil/GandG/wgs84/gravitymod/new_egm/new_egm.html 
23. Holmes SA, Pavlis NK (2007) Some aspects of harmonic analysis of data gridded on the ellipsoid. In Proceedings of the 1st International Symposium of the International Gravity Field Service - Gravity Field of the Earth. Harita Dergisi, Special Issue 18:151-156.

24. Huang J, Véronneau M, Pagiatakis SD (2003) On the ellipsoidal correction to the spherical Stokes solution of the gravimetric geoid. J Geod, 77:171-181, doi:10.1007/s001900-003-0317-6

25. Jekeli C (1988) The exact transformation between ellipsoidal and spherical harmonic expansions. manu geod, 13:106-113

26. Lelgemann D (1970) Untersuchungen zu einer genaueren Lösung des Problems von Stokes. Report C 155, Veröffentlichungen Deutsche Geodätische Kommission, Potsdam, Germany

27. Lemoine FG, Kenyon SC, Factor JK, Trimmer RG, Pavlis NK, Chinn DS, Cox CM, Klosko SM, Luthcke SB, Torrence MH, Wang YM, Williamson RG, Pavlis EC, Rapp RH, Olsen TR (1998) The development of the joint NASA GSFC and the National Imagery and Mapping Agency (NIMA) geopotential model EGM96. Technical report NASA/TP1998-206861, NASA, Washington, USA

28. Moritz H (1989) Advanced Physical Geodesy (2nd edition). Wichmann, Karlsruhe, Germany

29. Moritz H (2000) Geodetic Reference System 1980. J Geod, 74:128-140

30. Paul MK (1978) Recurrence relations for integrals of associated Legendre functions. Bull Geod, 52:177-190

31. Pavlis NK, Holmes SA, Kenyon SC, Factor JK (2012) The development and evaluation of the Earth Gravitational Model 2008 (EGM2008), J Geophys Res, 117:B04406, doi:10.1029/2011JB008916

32. Protter MH, Morrey CB (1964) Modern Mathematical Analysis. Addison-Wesley, Reading, USA

33. Rapp RH (1981) Ellipsoidal correction for geoid undulation computations using gravity anomalies in a cap. J Geophys Res, 86:10843-10848

34. Rapp RH, Pavlis NK (1990) The development and analysis of geopotential coefficient models to spherical harmonic degree 360. J Geophys Res, 95:21585-21911

35. Rummel R (1985) From the observational model to gravity parameter estimation. In: Schwarz KP (ed) Proceedings of the Beijing International Summer School: Local Gravity Field Approximation, University of Calgary, Calgary, Canada

36. Sebera J, Bouman J, Bosch W (2012) On computing ellipsoidal harmonics using Jekeli's renormalization. J Geod, 86:713-726, doi:10.1007/s00190-012-0549-4

37. Sjöberg LE (2003) The ellipsoidal corrections to order $e^{2}$ of geopotential coefficients and Stokes' formula. J Geod, 77:139-147, doi:10.1007/s00190-003-0321-x

38. Sjöberg LE, Featherstone WE (2004) Two-step procedures for hybrid geoid modelling. J Geod, 78:66-75, doi:10.1007/s00190-003-0367-9

39. Smith DA (1998) There is no such thing as "The" EGM96 geoid: Subtle points on the use of a global geopotential model, IGeS Bulletin, 8:17-28.

40. Stewart J (1995) Calculus (3rd edition). Brooks/Cole, Pacific Grove, USA

41. Vaníček P, Featherstone WE (1998) Performance of three types of Stokes's kernels in the combined solution for the geoid. J Geod, 72:684-697

42. Vaníček $\mathrm{P}$, Tenzer R, Sjöberg LE, Martinec Z, Featherstone WE (2004) New views of the spherical Bouguer gravity anomaly. Geophys J Int, 159:460-472, doi:10.1111/j.1365-246X.2004.02435.x 